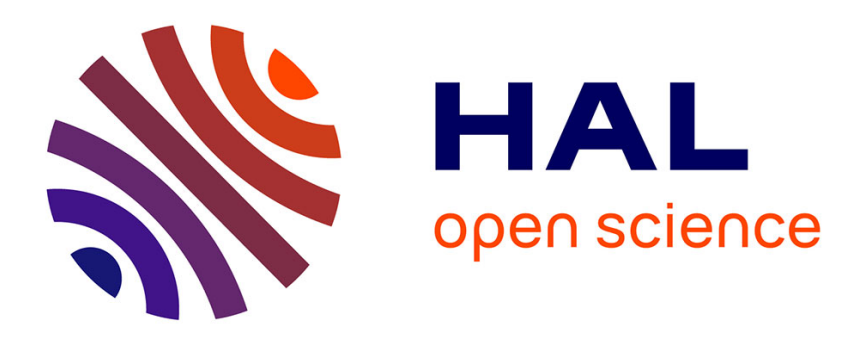

\title{
La sculpture grecque est-elle un objet d'histoire? À propos de deux ouvrages récents
}

Alain Duplouy

\section{To cite this version:}

Alain Duplouy. La sculpture grecque est-elle un objet d'histoire? À propos de deux ouvrages récents. L'Antiquité classique, 2005, 74 (1), pp.275-281. 10.3406/antiq.2005.2583 . halshs-00583883v2

\section{HAL Id: halshs-00583883 \\ https://shs.hal.science/halshs-00583883v2}

Submitted on 11 Dec 2019

HAL is a multi-disciplinary open access archive for the deposit and dissemination of scientific research documents, whether they are published or not. The documents may come from teaching and research institutions in France or abroad, or from public or private research centers.
L'archive ouverte pluridisciplinaire HAL, est destinée au dépôt et à la diffusion de documents scientifiques de niveau recherche, publiés ou non, émanant des établissements d'enseignement et de recherche français ou étrangers, des laboratoires publics ou privés. 


\section{La sculpture grecque est-elle un objet d'histoire ? À propos de deux} ouvrages récents

Alain Duplouy

\section{Citer ce document / Cite this document :}

Duplouy Alain. La sculpture grecque est-elle un objet d'histoire ? À propos de deux ouvrages récents. In: L'antiquité classique, Tome 74, 2005. pp. 275-281;

doi : 10.3406/antiq.2005.2583

http://www.persee.fr/doc/antiq_0770-2817_2005_num_74_1_2583

Document généré le 16/03/2016 


\section{La sculpture grecque est-elle un objet d'histoire ? À propos de deux ouvrages récents}

Nul ne niera que la sculpture archaïque offre un ensemble de documents généralement bien publiés - en regard d'autres vestiges matériels du passé -, qui constituent d'ordinaire le support d'un discours sur l'élaboration et l'évolution des formes. Catalogues de sites, recueils typologiques et synthèses retracent ainsi l'histoire d'un mode d'expression. Pour la plupart des spécialistes, la création artistique constitue un domaine autonome de la civilisation grecque et l'évolution des formes ne s'explique que par des lois qui lui sont propres. Aussi la sculpture grecque n'offre-telle souvent à l'histoire politique et sociale qu'une toile de fond culturelle. Pourtant, de par sa nature et son fonctionnement, la statuaire peut parfaitement être le support d'une enquête sur la société, apportant ainsi sa contribution à l'histoire des hommes. On ne rappellera jamais assez que la plupart des statues archaïques étaient soit votives soit funéraires et qu'en tant que telles, elles étaient tout d'abord pour les Grecs l'expression de commanditaires. À travers la statue, le dédicant avait le souci de se placer face aux autres et espérait retirer du miroir de la communauté l'image de sa position sociale. Dans l'intelligence de la sculpture archaïque, le regard des contemporains et la confrontation entre les diverses statues inscrites dans l'espace réduit du sanctuaire ou de la nécropole ne sont donc jamais des éléments secondaires. Dans un système où la statue fonctionne d'abord comme l'expression d'un commanditaire, et non pas comme une " simple » œuvre d'art, le rôle du sculpteur n'en était toutefois pas moins important. Celui-ci n'est nullement à exclure du schéma dont je viens de rappeler les principes; il en est au contraire un maillon essentiel. Dans le cas d'une commande spécifique, son rôle peut se concevoir comme celui d'un interprète qui a su mettre en forme, selon un savoir qui lui reste entièrement reconnu et avec son propre langage formel, les désirs et le projet de son client. Le savoir-faire de l'artiste pour ne pas dire son " génie " - demeure donc intact, mais il était à proprement parler mis au service du commanditaire et de ses ambitions sociales. En d'autres termes, l'existence et le développement de la sculpture archaïque dépendent d'acteurs sculpteurs et commanditaires - sur lesquels elle peut elle aussi, en retour, apporter sa part d'informations.

La parution récente de plusieurs ouvrages sur la sculpture grecque archaïque nous donne l'occasion de revenir ici sur certaines caractéristiques de la recherche actuelle. Nous rendrons compte ainsi de la synthèse collective dirigée par Peter C. Bol $^{1}$ et du recueil sur les korés de Katerina Karakasi, qui vient de connaître une

P. C. Bol et alii, Die Geschichte der antiken Bildhauerkunst. I. Frühgriechische Plastik. Mayence, Ph. von Zabern, 2002. 2 vol. 21,5 x $30 \mathrm{~cm}$. Textband : IX-342 p., 15 fig. Tafelband : V, 376 p., 366 fig. Prix : 75,80 €. ISBN 3-8053-2869-9. 
traduction anglaise après l'édition originale allemande ${ }^{2}$. Parce qu'il n'est pas possible d'évoquer ici toutes les œuvres discutées dans ces riches volumes, je prendrai essentiellement des exemples issus de la plastique ionienne, en particulier des cités de Samos et de Milet ${ }^{3}$.

1. - La typologie constitue l'un des piliers les plus fermement établis de la discipline. De fait, il est aisé de classer la sculpture archaïque en fonction des grands types statuaires que sont le kouros, la koré, la statue assise, la statue équestre, la caryatide... C'est ainsi que pour remplacer l'un des volumes de Gisela Richter, Katerina Karakasi a entrepris de recenser toutes les korés archaïques, y compris les statues fragmentaires. Pourquoi les korés serait-on tenté naïvement de dire ? À cette question l'auteur n'apporte malheureusement pas de réponse, ni dans le choix de son sujet ni dans le résultat de l'enquête. Ces statues ne sont pas exclusives dans les cités que l'auteur passe en revue, pas plus que leur distribution ne fait apparaitre quelque absence insolite. Si l'on peut donc se féliciter de disposer désormais d'un répertoire complet et à jour, on regrettera peut-être une absence de recul critique vis-à-vis du sujet, ainsi d'ailleurs qu'un certain maximalisme du catalogue. L'auteur inclut en effet parmi ses korés le torse du joueur d'aulos du monument sculpté par Généléos à Samos (S 13). Certes, Br. Freyer-Schauenburg le tenait jadis pour une koré, mais E. Walter-Karydi a clairement démontré depuis lors qu'il s'agissait en réalité d'un kouros drapés, qui n'a donc pas sa place dans le recueil.

Pareille sériation typologique est également adoptée par les auteurs de la synthèse dirigée par Peter Bol. Bien souvent kouroi et korés sont envisagés successivement, à travers des paragraphes distincts, sans qu'aucune relation ne soit établie entre les deux formes, ainsi pourvues d'histoires rigoureusement compartimentées. Un tel souci de classement frise néanmoins la caricature, lorsque Pavlina Karanastassis analyse séparément les statues du groupe familial offert par le Samien $-\imath \lambda \dot{\alpha} \rho \chi \eta \varsigma^{6}$ à l'Héraion de Samos, selon qu'il s'agit des korés (p. 186-187), de la statue assise (p. 197-198), du banqueteur (p. 200) ou du kouros drapé (p. 201). Pour se plier à une

2 K. KaraKasi, Archaische Koren. Munich, Hirmer Verlag, 2001. 1 vol. $24,5 \times 32 \mathrm{~cm}$. 472 p., 278 planches, dont 66 couleurs. 155€. ISBN 3-7774-8840-2. Archaic Korai. J. Paul Getty Museum, 2003. 1 vol. 24,5 x $32 \mathrm{~cm} .470$ p., 113 fig. couleurs, 675 fig. $\mathrm{n} / \mathrm{b} .125 \$$. ISBN 0-89236-699-0. C'est de l'édition originale allemande dont je rendrai compte ici.

3 Il s'agit en l'occurrence de sculptures dont j'ai repris l'étude dans ma thèse de doctorat Le prestige des élites. Recherches sur les modes de reconnaissance sociale en Grèce entre les $X^{\mathscr{e}}$ et $V^{\mathcal{E}}$ siècles (Université Libre de Bruxelles - Université Paris 1 Panthéon-Sorbonne, 2003), dont je prépare actuellement la publication.

4 Br. Freyer-Schauenburg, Samos XI. Bildwerke der archaische Zeit und des strengen Stils, Bonn, 1974, n $^{\circ} 13$.

5 E. WALTER-KaRYDI, "Geneleos", AthMitt 100 (1985), p. 91-95.

6 Notons que depuis G. DUNST, “Archaische Inschriften und Dokumente der Pentekon-

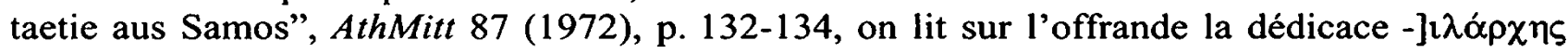

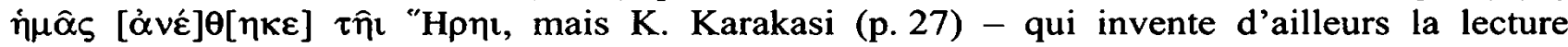

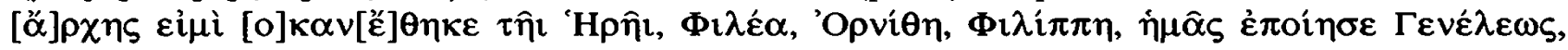
jamais proposée et tout à fait farfelue pour qui a vu l'offrande au Musée de Samos - et

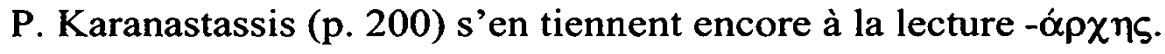


sacro-sainte typologie, l'analyse va en effet jusqu'à dissoudre l'unité de l'offrande, qui perd ainsi toute signification - et qui, à vrai dire, n'est plus comprise. Il n'est pas

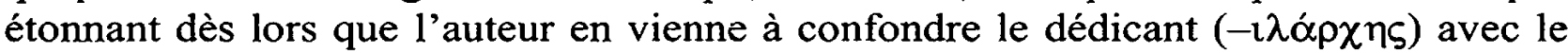
sculpteur (Généléos), puisqu'elle rebaptise un monument souvent appelé - à tort, et l'on comprendra pourquoi ! - «groupe de Généléos » en " Geneleos-Weihgeschenk » (p. 200), ce qui est sans doute le pire des contresens que l'on puisse faire en matière de sculpture grecque.

Si les historiens de l'art s'appuient sur des catégories typologiques pour justifier des études spécifiques, chez les Grecs c'était pourtant bien plus l'usage des statues qui déterminait leur appellation. En grec, les termes utilisés par les auteurs anciens ou rencontrés dans les inscriptions varient selon l'époque mais surtout selon la fonction de l'objet : à l'époque archaïque, nos kouroi et korés pouvaient donc être selon les cas votifs ou funéraires. La typologie des spécialistes contemporains recoupe donc assez mal les catégories conceptuelles des Anciens et risque ainsi d'obscurcir la compréhension des œuvres antiques dans leur contexte, bien plus souvent qu'elle n'apporte de réelles avancées épistémologiques.

Pourtant, il ne faut nullement jeter le bébé avec l'eau du bain : une typologie raisonnée est un instrument d'analyse incomparable, pour peu qu'elle serve à répondre à des questions précises. En l'occurrence, si kouroi et korés sont particulièrement répandus et si leur distribution respective n'apporte de commentaires qu'en relation avec la nature des sanctuaires, des types plus rares comme le kouros drapé ou le banqueteur aident à définir les spécificités de certains centres créateurs? La typologie devient alors une aide au service d'une problématique, bien plus qu'elle n'est une fin en elle-même.

2. - Le cadre topographique et géographique préoccupe de plus en plus les spécialistes ; et c'est une bonne chose. Le contexte de dédicace - dont les musées modernes et les cuvres "sans provenance » ont presque fait oublier l'existence constitue une composante essentielle du fonctionnement général des œuvres.

L'intérêt pour le lieu exact de découverte de chaque statue est fondamental et Karakasi a rassemblé à peu près toute la documentation disponible à ce sujet, résumant ses observations par des plans de site annotés. Mais en raison des nombreux remplois de ces statues comme matériaux de construction, le lieu de découverte n'indique généralement pas le lieu de dédicace initial de l'œuvre, ce qui est finalement la seule chose qui compte tant du point de vue du sculpteur que du dédicant. Un intérêt pour les bases de statues, que l'auteur ignore complètement, aurait certainement été plus révélateur, car les bases ont une tendance à moins bouger. C'est une habitude regrettable de la recherche actuelle que de dissocier la base, " simple bloc de marbre », de la statue, seul « véritable objet d'art », là où des savants comme Raubitschek et Jeffery avaient à juste titre insisté sur l'étroite parenté entre les deux éléments. L'excès est en revanche inverse chez D. Kreikenbom, dans le volume collectif dirigé par Peter Bol, lorsqu'il croit pouvoir écrire que les deux korés

7 Ainsi par exemple, pour le kouros drapé, type d'origine milésienne, B.A. BARLETTA, "The Draped Kouros Type and the Workshop of the Syracuse Youth", AJA 91 (1987), p. 233246. 
«jumelles » de Chéramyès, celle de Paris et celle de Samos, étaient « ursprünglich auf gemeinsamer Basis vereint " (p. 152). C'est là assurément une mauvaise lecture de la publication d'Helmut Kyrieleis, qui a clairement montré que cette base commune, certes creusée de deux cuvettes d'encastrement correspondant aux deux statues, ne renvoyait elle-même, pour diverses raisons, qu'à une phase de remploi, mais n'indiquait rien en ce qui concerne la disposition initiale des statues dans le sanctuaire $^{8}$, qui reste à découvrir. Quant aux statues assises milésiennes découvertes aux abords de Didymes, il est également faux d'écrire qu'elles devaient être, à l'origine, des offrandes consacrées dans le sanctuaire d'Apollon, bien avant d'être installées en remploi le long de la voie sacrée (p. 154). Depuis la découverte du sanctuaire de Kokkinólakka et la relecture attentive de la loi sacrée des Molpes, il est désormais indiscutable que la statue de Charès faisait partie d'un groupe de statues assises et que celles-ci étaient toutes dès l'origine installées sur le parcours de la procession annuelle allant de Milet à Didymes ${ }^{9}$, ce qui change bien évidemment l'interprétation générale, mais aussi la chronologie que l'on peut proposer à des statues dès lors contemporaines.

Quant à la géographie de la plastique archaïque, il faut bien constater que la démarche naguère développée par Ernst Langlotz ne rencontre guère d'écho dans ces deux ouvrages, en particulier dans le volume collectif dirigé par Peter Bol, qui n'a d'intérêt que pour la chronologie. Si Katerina Karakasi décide certes de répertorier les korés en fonction de leur lieu de découverte, c'est malheureusement un classement qui se veut commode, bien davantage qu'il ne sert réellement une problématique définie. L'auteur précise en effet d'emblée (p. 11-12) qu'elle ne s'intéressera pas aux questions de styles et d'écoles. L'ouvrage se présente ainsi comme une succession de monographies de site, qui bien souvent font double emploi avec les catalogues existants. Aucune étape n'est franchie dans la réflexion vers un classement géographique raisonné de la sculpture grecque archaïque. L'auteur pense à cet égard que la succession de ses chapitres reflète l'ordre chronologique d'apparition de la grande statuaire féminine en Grèce. Mais en commençant par Samos, Karakasi ne voit pas que les trois korés fragmentaires du $\mathrm{VII}^{\mathrm{e}}$ siècle $(\mathrm{S} 1,2$ et 3$)$ sont en réalité l'œuvre de sculpteurs naxiens, venus à Samos à l'appel de commanditaires locaux ${ }^{10}$. Rares sont en effet les sanctuaires ou les cités de Grèce à présenter un faciès stylistique homogène. Bien au contraire, chaque ensemble votif apparaît souvent comme un assemblage plus ou moins hétéroclite d'objets aux formes diverses. Si l'on tient à opérer un classement géographique de la plastique archaïque, il convient avant tout,

8 H. KYRIELEIS, "Eine neue Kore des Cheramyes", AntPlast 24 (1995), p. 15-19.

9 Cf. Kl. TuCHELT, Didyma III 1. Ein Kultbezirk an der Heiligen Strasse von Milet nach Didyma, Mayence, 1996, p. 233 n. 211.

10 Fr. CROISSANT, "Observations sur la naissance et le développement des styles dans la

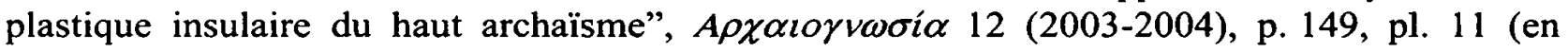
grec); Les premières korés cycladiques, dans J. Kouralos et Fr. Prost (éd.), La sculpture des Cyclades à l'époque archaïque. Histoire des ateliers, rayonnement des styles. Actes du colloque d'Athènes, 7-9 septembre 1998, à paraître. 
comme le soulignait Jean Ducat" ${ }^{11}$ d'accorder une grande importance à distinguer, dans le matériel d'un sanctuaire ou d'une cité, les créations locales des "importations ». La circulation des ateliers de sculpteurs dans l'espace égéen est aujourd'hui une chose bien connue et bien documentée ${ }^{12}$, qui rend aisément compte de la bigarrure formelle de certaines collections de statues. C'est là malheureusement une problématique historique qui échappe largement aux auteurs de ces deux volumes.

3. - La chronologie est incontestablement l'obsession principale de bon nombre de spécialistes de sculpture, qui tentent d'affiner à chaque découverte la chronologie de l'ensemble de la série. Alors que les repères absolus en matière de plastique archaïque se comptent sur les doigts d'une main, l'extraordinaire précision qu'il s'agisse de chronologie relative ou absolue - dont font preuve certains ouvrages laisse assurément rêveur. C'est en tout cas l'impression que donnent au lecteur les tableaux chronologiques dressés en fin de volume par Katerina Karakasi. On sait pourtant l'extrême fragilité des postulats évolutionnistes de Gisela Richter, qui s'inscrivent en fait dans un raisonnement circulaire : il faudrait s'assurer au préalable d'une chronologie détaillée des œuvres, pour être certain que leur évolution tend bien vers une représentation de plus en plus naturaliste du corps humain; or, c'est précisément ce seul présupposé qui régit l'ensemble du processus de datation.

Assuré d'une chronologie fine, Peter Bol a cru quant à lui pouvoir dresser un bilan de la production statuaire génération par génération. Ainsi l'époque archaïque se trouve-t-elle divisée en six phases au moins, chacune confiée à l'expertise d'un collaborateur différent: der orientalisierende Stil (Michael Maaß), dädalische Plastik (Gabriele Kaminski), früharchaische Plastik (Christiane Vorster), reifarchaische Plastik (Detlev Kreikenbom), hocharchaische Plastik (Pavlina Karanastassis), spätarchaische Plastik (Caterina Maderna-Lauter). Ce "saucissonnage» de la plastique archaïque, doublé d'un éclatement des perspectives entre des auteurs aux préoccupations et sensibilités diverses, est extrêmement préjudiciable à l'interprétation historique des œuvres, autant d'ailleurs qu'à une étude de leur évolution formelle. Alors que la nécropole et le sanctuaire ont précisément pour fonction d'inscrire dans la durée les statues et d'en faire des marqueurs permanents pour le visiteur ou le futur commanditaire, la production statuaire est ici atomisée et ainsi réduite en particules élémentaires disjointes de tout ce qui contribue à leur donner un sens.

Dans l'établissement d'une chronologie détaillée de la plastique archaïque, il est bien entendu nécessaire d'accorder le plus grand intérêt aux données de la fouille, lorsqu'elles sont disponibles et signifiantes. Il n'est dès lors guère compréhensible, compte tenu de l'importance de la chronologie dans l'organisation générale du volume, que Detlev Kreikenbom (p. 153-154) ne souffle mot du contexte de décou-

1 J. DuCAT, Les kouroi du Ptoion. Le sanctuaire d'Apollon Ptoieus à l'époque archaïque, Paris, 1971, p. 100 notait «Démêler, dans la sculpture trouvée à Perdikovrysi, ce qui est "local" de ce qui est "importé" semble donc devoir être un des buts principaux de la présente recherche " .

12 Voir notamment D. VIVIERS, Le bouclier signé du trésor de Siphnos à Delphes: "Régions stylistiques » et ateliers itinérants ou la sculpture archaïque face aux lois du marché, dans Chr. Müller et Fr. Prost (éd.), Identités et cultures dans le monde méditerranéen antique, Paris, 2002, p. 53-85. 
verte du kouros d'Ischès, alors que la fouille a clairement établi la situation stratigraphique de sa base. La construction de celle-ci est en effet assurément antérieure au deuxième quart du $\mathrm{VI}^{\mathrm{e}}$ siècle $^{13}$. Base et colosse formaient ainsi un monument qui se dressait dès le premier quart du $\mathrm{VI}^{\mathrm{e}}$ siècle le long de la voie sacrée. Son appartenance à l'« archaïsme mature » (reifarchaische Plastik), pour reprendre la périodisation de Peter Bol, n'est donc pas à remettre en cause, mais c'en est à tout le moins l'un des seuls points de repère assurés, ce qui aurait mérité d'être dit.

Enfin, rappelons qu'il n'est guère possible d'établir une chronologie relative des œuvres sans distinguer au préalable entre les divers centres créateurs. On ne compare que ce qui est comparable et il n'est guère fécond par exemple de confronter $\grave{a}$ des fins chronologiques un kouros béotien avec une statue samienne ou naxienne. Malgré des tables compilées par cité, la chronologie régionale de Karakasi ne tient que très partiellement compte de ce principe, dans la mesure où, comme nous l'avons vu, l'auteur n'opère pas de distinction entre les productions locales et les « importations ».

4. - L'illustration du volume de Katerina Karakasi, enfin, est somptueuse. Même les planches en couleurs sont ici les bienvenues, car elles montrent clairement les traces de polychromie que certaines de ces statues ont conservées. Plus encore que les Korai de Richter (dans leur édition originale de 1968, non dans l'anastatique de 1988), les Archaische Koren de Karakasi - autant d'ailleurs que la traduction anglaise - sont appelées à devenir un répertoire photographique de référence, pour l'exhaustivité et la qualité des images reproduites. Le choix d'un épais papier brillant pour le volume de Peter Bol est sans doute moins judicieux, car il provoque des reflets désagréables, mais la qualité des clichés est dans l'ensemble - si l'on excepte quelques problèmes de contraste - tout aussi bonne, autant qu'il est agréable de disposer d'un volume de figures distinct du texte.

En revanche, il convient de dénoncer l'absence totale de composition des planches. Pour des volumes qui ne se veulent pas des catalogues de sites ou de musées, mais bien des synthèses ayant pour ambition d'inscrire les statues dans un discours artistique ou historique, il est regrettable que l'image ne soit pas mise au service de certaines démonstrations. L'analyse de la statuaire antique procède normalement par comparaison ou par opposition des œuvres. Ce n'est jamais le cas ici ; chaque statue est généralement isolée des autres sur une pleine page et rares sont les relations suggérées par l'image. Ainsi les auteurs se privent-ils de l'une des preuves les plus convaincantes à l'appui de leur argumentation, mais aussi tout simplement d'un extraordinaire élément de réflexion. Par exemple, dans l'ouvrage dirigé par Peter Bol, si les illustrations du colosse d'Ischès (Abb. 229) et du kouros Samos-Istanbul (Abb. 258) sont séparées de quelque vingt pages, c'est parce que les deux statues sont traitées par deux auteurs différents et, partant, assignées à des périodes distinctes de la production statuaire samienne. Un rapprochement des deux kouroi, comme l'avait

13 H.J. KIENAST, “Ausgrabungen im Heraion von Samos 1980/81”, $A A$ (1985), p. 378383 ; Samos X. Der grosse Kuros von Samos, Bonn, 1996, p. 7-15, 48-49. 
d'ailleurs suggéré Helmut Kyrieleis ${ }^{14}$, aurait pourtant permis de reconnaître une parenté formelle évidente entre les deux statues, mais aussi d'établir entre elles un rapport de proportion particulier. Or ces deux faits obligent non seulement à remonter la date du kouros Samos-Istanbul, mais aussi à y voir une statue issue du même atelier que le colosse d'Ischès.

Pour peu que l'on ne s'attache pas seulement à leur statut d'œuvres d'art, les statues archaïques, à travers les relations qu'elles entretenaient avec les hommes qui les ont créées et ceux qui les avaient commandées, constituent une documentation de premier ordre, dont les historiens ont souvent méconnu l'intérêt. À ce titre, la sculpture grecque est incontestablement l'objet d'une histoire, qui n'est pas seulement celle des formes, mais aussi celle des hommes. Encore convient-il de formuler les problématiques adéquates et de se doter des outils d'analyse appropriés à la nature et au fonctionnement spécifiques de cette abondante documentation archaïque. À l'heure où les archéologues ont montré qu'il était possible d'élaborer un discours historique à partir d'objets dépourvus de toute ambition artistique, la sculpture grecque demeure souvent à l'écart des problématiques d'histoire sociale, comme si son statut d'œuvre d'art la préservait de toute interprétation contextuelle. Les deux lectures, artistique et historique, ne sont pourtant pas exclusives, mais fort heureusement complémentaires : l'évolution formelle de la sculpture grecque et sa diversité stylistique ne seraient-elles pas surtout le résultat d'une compétition sans cesse renouvelée ayant poussé les artistes et leur clientèle à la recherche de formes originales? Aussi est-il manifeste, tant en ce qui concerne l'histoire des hommes que celle des formes, que les ouvrages de Peter Bol et de Katerina Karakasi perpétuent une tradition d'étude stérile, qu'il convient de dénoncer avec vigueur.

\section{Alain DUPLOUY}

14 H. Kyrieleis, Samos X, p. 49-51. Cf. A. DuplouY, "L'atelier du kouros d'Ischès. Observations sur l'organisation de la production statuaire en Ionie archaïque", $R E A 108$ (2006), sous presse. 